\title{
ELASTIC-PLASTIC ANALYSIS OF PRESSURE VESSELS AND ROTATING DISKS MADE OF FUNCTIONALLY GRADED MATERIALS USING THE ISOGEOMETRIC APPROACH
}

\author{
Amir T. Kalali, Behrooz Hassani, Saied Hadidi-Moud \\ Mechanical Engineering Department, Ferdowsi University of Mashhad, Iran \\ e-mail:amir.kalali.121@stu-mail.um.ac.ir; b_hassani@um.ac.ir; hadidi@um.ac.ir
}

\begin{abstract}
An NURBS-based isogeometric analysis for elastic-plastic stress in a cylindrical pressure vessel is presented. The vessel is made of a ceramic/metal functionally graded material, i.e. a particle-reinforced composite. It is assumed that the material plastic deformation follows an isotropic strain-hardening rule based on the von Mises yield criterion. The mechanical properties of the graded material are modelled by the modified rule of mixtures. Selected finite element results are also presented to establish the supporting evidence for validation of the isogeometric analysis. Similar analyses are performed and solutions for spherical pressure vessel and rotating disk made of FGMs are also provided.
\end{abstract}

Keywords: isogeometric analysis, NURBS, functionally graded material, modified rule of mixtures

\section{Introduction}

The intensity and variation of stress distributions due to large mismatch in material properties can be substantially reduced if micro-structural transition behaviour, i.e. a graded material model, is used. Advances in material synthesis technologies have spurred the development of functionally graded materials (FGM) with promising applications in aerospace, transportation, energy, cutting tools, electronics, and biomedical engineering (Chakraborty et al., 2003). An FGM comprises a multi-phase material with volume fractions of the constituents varying gradually in a predetermined profile, thus yielding a non-uniform microstructure in the material with continuously graded properties (Jin et al., 2003).

Elastic and elastic-plastic analyses of thick-walled pressure vessels have always attracted a lot of research interest because of their importance in engineering applications. Figueiredo et al. (2008) proposed a numerical methodology in order to predict the elastic-plastic stress behaviour of functionally graded cylindrical vessels subjected to internal pressure. It was assumed that the structures undergo small strain and that the material properties of the graded layer were modelled by the modified rule of mixtures approximation. Furthermore, the plastic domain for ductile phases was defined through the von Mises yield criterion. They proposed an iterative method for solving the nonlinear system combining a finite element approximation and an incremental-iterative scheme. Haghpanah Jahromi et al. (2009, 2010) extended the Variable Material Property (VMP) method developed by Jahed and Dubey (1997) for materials with varying elastic and plastic properties. In the VMP method, the linear elastic solution to the boundary value problem was used as a basis to generate the inelastic solution. Through iterative analysis, the VMP method was used to obtain the distribution of material parameters which were considered as field variables. The application of the VMP method, generally applied to homogeneous elastic-plastic materials (Jahed and Shirazi, 2001; Jahed et al., 2005, 2006), was extended to materials with varying elastic-plastic properties in order to calculate the residual stresses in an autofrettaged FGM cylindrical vessel. 
Although there are several papers on the elastic analysis of FGM spherical pressure vessels in the literature (You et al., 2005; Dai et al., 2006; Chen and Lin, 2008), elastic-plastic stress analysis of FGM spherical pressure vessels is not such a customary study. Sadeghian and Ekhteraei (2011) studied thermal stress field for an FGM spherical pressure vessel made of an elastic-perfectly plastic and a power law material model.

Similar to the FGM cylindrical and spherical vessels, much of the studies on FGM rotating disks has been carried out in elasticity cases (Durodola and Attia, 2000; Bayat et al., 2008). Haghpanah Jahromi et al. (2012) applied the VMP method to estimate the elasto-plastic stresses in a rotating disk with varying elastic and plastic properties in the radial direction.

In this paper, isogeometric analysis is proposed for predicting stress components of a strain-hardening cylinder based on the von Mises yield criterion under plane stress conditions. Isogeometric analysis was introduced by Hughes et al. (2005) as a generalisation of the standard finite element analysis. In isogeometric analysis, the solution space for dependent variables is represented in terms of the same functions which represent the geometry. The geometric representation is typically smooth, whereas the solution space for the standard finite element analysis is continuous but not smooth. Adoption of the isogeometric concept has shown computational advantages over the standard finite element analysis in terms of accuracy and analysis time in many application areas, including solid and structural mechanics. Most CAD systems use spline basis functions and often Non-Uniform Rational B-Splines (NURBS) of different polynomial orders to represent geometry. Results obtained from finite element analysis using the commercial software ABAQUS (v. 6.10) were used to validate the results from the isogeometric analysis. The analysis was further extended to obtain solutions for FGM spherical vessels and rotating disks.

A brief review of the isogeometric analysis based on NURBS is presented in Section 2. This is followed in Section 3 by describing the details of isogeometric analysis formulation for elastic-plastic cases (functionally graded cylindrical and spherical vessels and rotating disks). In Section 4, we describe material properties of the graded layer modelled by the modified rule of mixtures, whereas in Section 5 the results of elastic-plastic analyses are presented. Finally, in Section 6, key conclusions are pointed out.

\section{Fundamentals of NURBS-based isogeometric analysis}

\subsection{B-splines and NURBS}

Non-uniform rational B-splines (NURBS) are a standard tool for describing and modelling curves and surfaces in the computer aided design and computer graphics.

B-splines are piecewise polynomial curves composed of linear combinations of B-spline basis functions. The piecewise definition allows approximation of a large number of control points using lower order polynomials. The coefficients are points in space, referred to as the control points. A knot vector $\boldsymbol{\Xi}$ is a set of non-decreasing real numbers representing coordinates in the parametric space of the curve

$$
\boldsymbol{\Xi}=\left[\xi_{1}, \xi_{2}, \xi_{3}, \ldots, \xi_{i}, \ldots, \xi_{n+p+1}\right]
$$

where $p$ is the order of the $\mathrm{B}$-spline and $n$ refers to the number of the basis functions (also control points). The interval $\left[\xi_{1}, \xi_{n+p+1}\right]$ is called a patch.

The B-spline basis functions for a given degree $p$ are defined recursively over the parametric domain by the knot vector. The piecewise constants are first defined as

$$
N_{i, 0}(\xi)= \begin{cases}1 & \text { if } \xi_{i} \leqslant \xi<\xi_{i+1} \\ 0 & \text { otherwise }\end{cases}
$$


For $p>0$, the basis functions are defined by the following recursion formula

$$
N_{i, p}(\xi)=\frac{\xi-\xi_{i}}{\xi_{i+p}-\xi_{i}} N_{i, p-1}(\xi)+\frac{\xi_{i+p+1}-\xi}{\xi_{i+p+1}-\xi_{i+1}} N_{i+1, p-1}(\xi)
$$

A B-spline surface is constructed by the basis functions in two directions, $N_{i, p}(\xi)$ and $M_{j, q}(\eta)$, and a set of control points $P_{i j}, i=1,2, \ldots, n, j=1,2, \ldots, m$. Similar to the first parametric direction $\xi, M_{j, q}(\eta)$ is also defined by Eqs. (2.2) and (2.3), but another knot vector $\mathbf{H}=\left[\eta_{1}, \eta_{2}, \eta_{3}, \ldots, \eta_{j}, \ldots, \eta_{m+q+1}\right]$ constitutes the foundation. Often, the B-spline order is the same in both directions, i.e. $p=q$.

The surface is to be drawn in the two-dimensional space, $P_{i j} \in R^{2}$. The B-spline surface is then interpolated by

$$
S(\xi, \eta)=\sum_{i=1}^{n} \sum_{j=1}^{m} N_{i, p}(\xi) M_{j, q}(\eta) P_{i j}
$$

The B-spline surface is the result of a tensor product. The patch for the surface is now the domain $\left[\xi_{1}, \xi_{n+p+1}\right] \times\left[\eta_{1}, \eta_{n+p+1}\right]$. Identifying the logical coordinates $(i, j)$ of the B-spline surface with the traditional notation of the node $A$ and the Cartesian product of the associated basis functions with the shape function $N_{A}(\xi, \eta)=N_{i, p}(\xi) M_{j, q}(\eta)$, the familiar finite element notation is recovered, namely

$$
S(\xi, \eta)=\sum_{A=1}^{n m} N_{A}(\xi, \eta) P_{A}
$$

B-splines are non-rational functions that form non-rational B-spline curves and surfaces. A rational curve or surface can represent conical sections in an exact manner. Non-uniform rational B-splines (NURBS) are therefore introduced by including weights on the control points. The NURBS basis functions will differ from the B-spline basis functions, but the knot vectors, the tensor product nature, and refinement mechanisms are unchanged.

The NURBS surface is given by

$$
S(\xi, \eta)=\frac{1}{w(\xi, \eta)} \sum_{A=1}^{n m} N_{A}(\xi, \eta) w_{A} P_{A}=\sum_{A=1}^{n m} \bar{N}_{A}(\xi, \eta) P_{A}
$$

where

$$
w(\xi, \eta)=\sum_{A=1}^{n m} N_{A}(\xi, \eta) w_{A} \quad \bar{N}_{A}(\xi, \eta)=\frac{N_{A}(\xi, \eta) w_{A}}{w(\xi, \eta)}
$$

\subsection{Fundamentals of the isogeometric analysis}

The isogeometric analysis was defined by Hughes et al. (2005) and means that the analysis model uses the same mathematical description as the geometry model. This notion of using the same basis for geometry and analysis is called the isoparametric concept, and it is quite common in the classical finite element analysis. The fundamental difference between the isogeometric analysis and the finite element analysis is that, in the FEA, the basis chosen for the approximation of the unknown solution fields is used to approximate known geometry whereas the isogeometric analysis turns this idea around and selects a basis capable of exactly representing the known geometry, and uses it as a basis for the fields we wish to approximate (Cottrell et al., 2009).

The main advantages of the isogeometrical method, compared to other numerical methods, can be summarised as below:

- feduction in size of the system of equations, 
- flexibility and accuracy in the definition of geometry and its boundaries,

- the possibility of keeping the original model in the whole process without several remeshing in problems with a varying domain of interest,

- considerable ease in implementing adaptively and mesh refinement,

- accuracy in satisfaction of the essential boundary conditions,

- applicability of the method in problems of functionally graded materials (Hassani et al., 2011).

And the main disadvantages of the isogeometric analysis can be mentioned as in the following:

- the control points of geometry commonly are not a part of the physical domain of the problem,

- the relative difficulty of establishing a correspondence between the point in the domain and the solution.

\section{Isogeometric analysis formulation for the elastic-plastic case}

\subsection{Elastic formulation}

We use the principle of virtual displacement applied to a plane elastic body

$$
0=\int_{V}\left(\sigma_{i j} \delta \varepsilon_{i j}+\rho \ddot{u}_{i} \delta u_{i}\right) d V-\int_{V} f_{i} \delta u_{i} d V-\oint_{S} t_{i} \delta u_{i} d s
$$

where $\sigma_{i j}$ is the Cauchy stress, $\varepsilon_{i j}$ is strain, $\rho$ is density, $\ddot{u}_{i}$ is the acceleration component, $u_{i}$ is the displacement component (i.e. $u, v, w), f_{i}$ is the body force component, $t_{i}$ is the traction component (i.e. $t_{x}, t_{y}, t_{z}$ ), $V$ is volume and $S$ is surface area corresponding to the volume.

In the cylindrical coordinate system and axisymmetric condition, Eq. (3.1) can be rewritten as follows

$$
0=2 \pi \iint\left(\sigma_{i j} \delta \varepsilon_{i j}+\rho \ddot{u}_{i} \delta u_{i}\right) r d r d z-2 \pi \iint f_{i} \delta u_{i} r d r-\oint_{S} t_{i} \delta u_{i} d s
$$

By using the NURBS basis functions, the approximated displacement functions can be written as

$$
\begin{aligned}
& r(\xi, \eta)=\sum_{A=1}^{n m} N_{A}(\xi, \eta) r_{A}=\left[N_{1}(\xi, \eta), N_{2}(\xi, \eta), \ldots, N_{n m}(\xi, \eta)\right]\left[\begin{array}{c}
r_{1} \\
r_{2} \\
\vdots \\
r_{n m}
\end{array}\right]=\mathbf{N r} \\
& z(\xi, \eta)=\sum_{A=1}^{n m} N_{A}(\xi, \eta) z_{A}=\mathbf{N z} \\
& u(\xi, \eta)=\sum_{A=1}^{n m} N_{A}(\xi, \eta) u_{A}=\mathbf{N u} \quad w(\xi, \eta)=\sum_{A=1}^{n m} N_{A}(\xi, \eta) w_{A}=\mathbf{N} \mathbf{w} \\
& \delta u(\xi, \eta)=\sum_{A=1}^{n m} N_{A}(\xi, \eta) \delta u_{A}=\mathbf{N} \boldsymbol{\delta} \mathbf{u} \\
& \quad \delta w(\xi, \eta)=\sum_{A=1}^{n m} N_{A}(\xi, \eta) \delta w_{A}=\mathbf{N} \boldsymbol{\delta} \mathbf{w}
\end{aligned}
$$

where $r_{A}$ and $z_{A}$ are the $x$ - and $y$-coordinates of the control points of the surface, $u_{A}$ and $w_{A}$ are the control points of the displacement. 
The stress and strain relationships are given by

$$
\boldsymbol{\varepsilon}=\mathbf{T N}\left[\begin{array}{l}
u \\
w
\end{array}\right]=\mathbf{B}\left[\begin{array}{l}
u \\
w
\end{array}\right] \quad \boldsymbol{\sigma}=\mathbf{C} \boldsymbol{\varepsilon}=\mathbf{C B}\left[\begin{array}{c}
u \\
w
\end{array}\right]
$$

where $\mathbf{T}$ is the matrix of differential operators, $\mathbf{C}$ is the constitutive matrix (constitutive matrix is calculated via Young's modulus and Poisson's ratio) and $\mathbf{B}=\mathbf{T N}$. In this study it is assumed that Poisson's ratio $\nu$ is a material constant while Young's modulus $E(r)$ varies with the position across the wall thickness of the vessel (disk).

Substituting Eqs. (3.3) and (3.4) into Eq. (3.2), and in the absence of inertia forces, we obtain

$$
0=2 \pi \iint\left(\mathbf{B}^{\mathrm{T}} \mathbf{C B}\left[\begin{array}{c}
u \\
w
\end{array}\right]\right) r d r d z-2 \pi \iint \mathbf{N}^{\mathrm{T}}\left[\begin{array}{l}
f_{r} \\
f_{z}
\end{array}\right] r d r d z-\oint_{S} \mathbf{N}^{\mathrm{T}}\left[\begin{array}{l}
t_{r} \\
t_{z}
\end{array}\right] d s
$$

Note that in Eq. (3.5) all variables are written in terms of the parameters $\xi$ and $\eta$ which is similar to mapping in the standard finite element method where the base or unit elements are used

$$
\begin{aligned}
0= & 2 \pi \int_{\xi_{1}}^{\xi_{n+p+1}} \int_{\eta_{1}}^{\eta_{m+q+1}} \mathbf{B}^{\mathrm{T}} \mathbf{C B}\left[\begin{array}{l}
u \\
w
\end{array}\right] r(\operatorname{det} \mathbf{J}) d \eta d \xi \\
& -2 \pi \int_{\xi_{1}}^{\xi_{n+p+1}} \int_{\eta_{1}}^{\eta_{m+q+1}} \mathbf{N}^{\mathrm{T}}\left[\begin{array}{l}
f_{r} \\
f_{z}
\end{array}\right] r(\operatorname{det} \mathbf{J}) d \eta d \xi-\oint_{S} \mathbf{N}^{\mathrm{T}}\left[\begin{array}{c}
t_{r} \\
t_{z}
\end{array}\right] d s
\end{aligned}
$$

where

$$
\mathbf{J}=\left[\begin{array}{ll}
\partial r / \partial \xi & \partial z / \partial \xi \\
\partial r / \partial \eta & \partial z / \partial \eta
\end{array}\right]
$$

and the matrix form is as follows

$$
\mathbf{K u}=\mathbf{F}+\mathbf{T}
$$

where

$$
\begin{aligned}
& \mathbf{K}=2 \pi \int_{\xi_{1}}^{\xi_{n+p+1}} \int_{\eta_{1}}^{\eta_{m+q+1}} \mathbf{B}^{\mathrm{T}} \mathbf{C B}\left[\begin{array}{l}
u \\
w
\end{array}\right] r(\operatorname{det} \mathbf{J}) d \eta d \xi \\
& \mathbf{F}=2 \pi \int_{\xi_{1}}^{\xi_{n+p+1}} \int_{\eta_{1}}^{\eta_{m+q+1}} \mathbf{N}^{\mathrm{T}}\left[\begin{array}{l}
f_{r} \\
f_{z}
\end{array}\right] r(\operatorname{det} \mathbf{J}) d \eta d \xi \\
& \mathbf{T}=\oint_{S} \mathbf{N}^{\mathrm{T}}\left[\begin{array}{c}
t_{r} \\
t_{z}
\end{array}\right] d s
\end{aligned}
$$

Integrals in Eq. (3.8) can be calculated using the Gauss-Legendre method of numerical integration.

In order to obtain stress distributions for a spherical thick-walled functionally graded pressure vessel, the isogeometric analysis formulation is rewritten in the spherical coordinate system $(r, \theta, \varphi)$. 


\subsection{Plastic formulation}

In order to formulate a theory which models elasto-plastic material deformation, three requirements have to be met:

- before the onset of plastic deformation, an explicit relationship between stress and strain must be formulated to describe material behaviour under elastic conditions,

- a yield criterion must be postulated to indicate the stress level at which plastic flow commences,

- a relationship between stress and strain must be developed for post yield behaviour, when the deformation is made up of both elastic and plastic components (Owen and Hinton, 1980).

According to the normality hypothesis of plasticity, the plastic strain increment $d \varepsilon^{p}$ is defined as:

$$
d \varepsilon^{p}=d \lambda \frac{\partial Q}{\partial \sigma}
$$

where $Q$ is the yield function and $d \lambda$ is called the plastic multiplier.

Assuming that the material plastic deformation follows the isotropic strain-hardening rule based on the von Mises yield criterion $\mathbf{n} \equiv \partial Q / \partial \sigma=\left[3 /\left(2 \sigma_{e}\right)\right] \mathbf{S}$ and $d \lambda=d \varepsilon_{e}^{p}$, Eq. (3.9) may be rewritten as

$$
\mathbf{d} \varepsilon^{p}=\frac{3}{2} \frac{d \varepsilon_{e}^{p}}{\sigma_{e}} \mathbf{S}
$$

where $d \varepsilon_{e}^{p}$ is the equivalent plastic strain increment. The superscripts $p$ and $e$ denote plasticity and elasticity conditions respectively, also the subscript $e$ denotes equivalent (effective) parameters (stress or plastic strain).

The equivalent stress $\sigma_{e}$ and the deviatoric stress $S$ for the plane stress field are defined as

$$
\sigma_{e}=\sqrt{\sigma_{r}^{2}+\sigma_{\theta}^{2}-\sigma_{r} \sigma_{\theta}} \quad \mathbf{S}=\left[\begin{array}{c}
S_{r} \\
S_{\theta} \\
S_{z}
\end{array}\right]=\left[\begin{array}{c}
\frac{2 \sigma_{r}-\sigma_{\theta}}{3} \\
\frac{2 \sigma_{\theta}-\sigma_{r}}{3} \\
-\frac{\sigma_{r}+\sigma_{\theta}}{3}
\end{array}\right]
$$

where $\sigma_{r}$ and $\sigma_{\theta}$ are the radial and hoop stresses, respectively. For a linear strain hardening material (Fig. 1), the yield stress $\sigma_{y}$ and the plastic multiplier $d \lambda$ are determined by

$$
\sigma_{y}=\sigma_{y 0}(r)+h_{p}(r) \varepsilon_{e}^{p} \quad d \lambda=\frac{\mathbf{n}^{\mathrm{T}} \mathbf{C d} \varepsilon}{\mathbf{n}^{\mathrm{T}} \mathbf{C n}+h_{p}}
$$

where $h_{p}(r)$ is the plasticity modulus (i.e. the gradient of the stress-plastic strain curve) and $\sigma_{y 0}(r)$ is the initial yield stress of the FGM material. Both $h_{p}(r)$ and $\sigma_{y 0}(r)$ are functions dependent on the radial position $r$. The stress increment is given by

$$
\mathbf{d} \boldsymbol{\sigma}=\mathbf{C d} \varepsilon^{e}=\mathbf{C}\left(\mathbf{d} \varepsilon-\mathbf{d} \varepsilon^{p}\right)=\mathbf{C}(\mathbf{d} \varepsilon-d \lambda \mathbf{n})
$$

by substituting Eq. (3.12) 2 into Eq. (3.13), we can obtain the complete elasto-plastic incremental stress-strain relation

$$
\mathbf{d} \boldsymbol{\sigma}=\mathbf{C}^{e p} \mathbf{d} \varepsilon \quad \mathbf{C}^{e p}=\mathbf{C}-\frac{\mathbf{C n n}^{\mathrm{T}} \mathbf{C}}{\mathbf{n}^{\mathrm{T}} \mathbf{C n}+h_{p}}
$$

where the superscripts ep denote the elasto-plastic behaviour. 


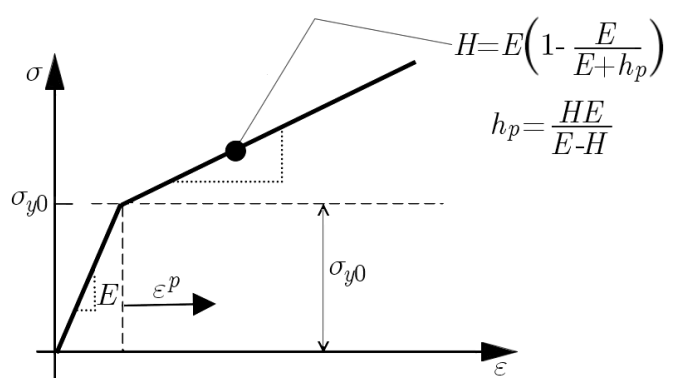

Fig. 1. Stress-strain curve for linear strain hardening

If we denote all quantities at the iteration $k$ with a superscript $k$, and those at the next increment by $k+1$ in a similar way, then we may write

$$
d \lambda^{k}=\frac{\mathbf{n}^{k^{\mathrm{T}}} \mathbf{C d} \boldsymbol{\varepsilon}^{k}}{\mathbf{n}^{k^{\mathrm{T}}} \mathbf{C n}^{k}+h_{p}} \quad \mathbf{d} \boldsymbol{\sigma}^{k}=\mathbf{C}\left(\mathbf{d} \varepsilon^{k}-d \lambda^{k} \mathbf{n}^{k}\right)
$$

The integration to obtain the quantity at the end of the time step $\Delta t$ may be then written as

$$
\boldsymbol{\sigma}^{k+1}=\boldsymbol{\sigma}^{k}+\mathbf{d} \boldsymbol{\sigma}^{k}
$$

If relatively large load increments are to be permitted, the process described can lead to an inaccurate prediction of the stresses. Two parameters $R$ (reduction factor) and $m$ (the excess of the yield stress is divided into $m$ parts) can help to minimize the error (Owen and Hinton, 1980). The algorithm for plasticity isogeometric analysis is summarized in Fig. 2.

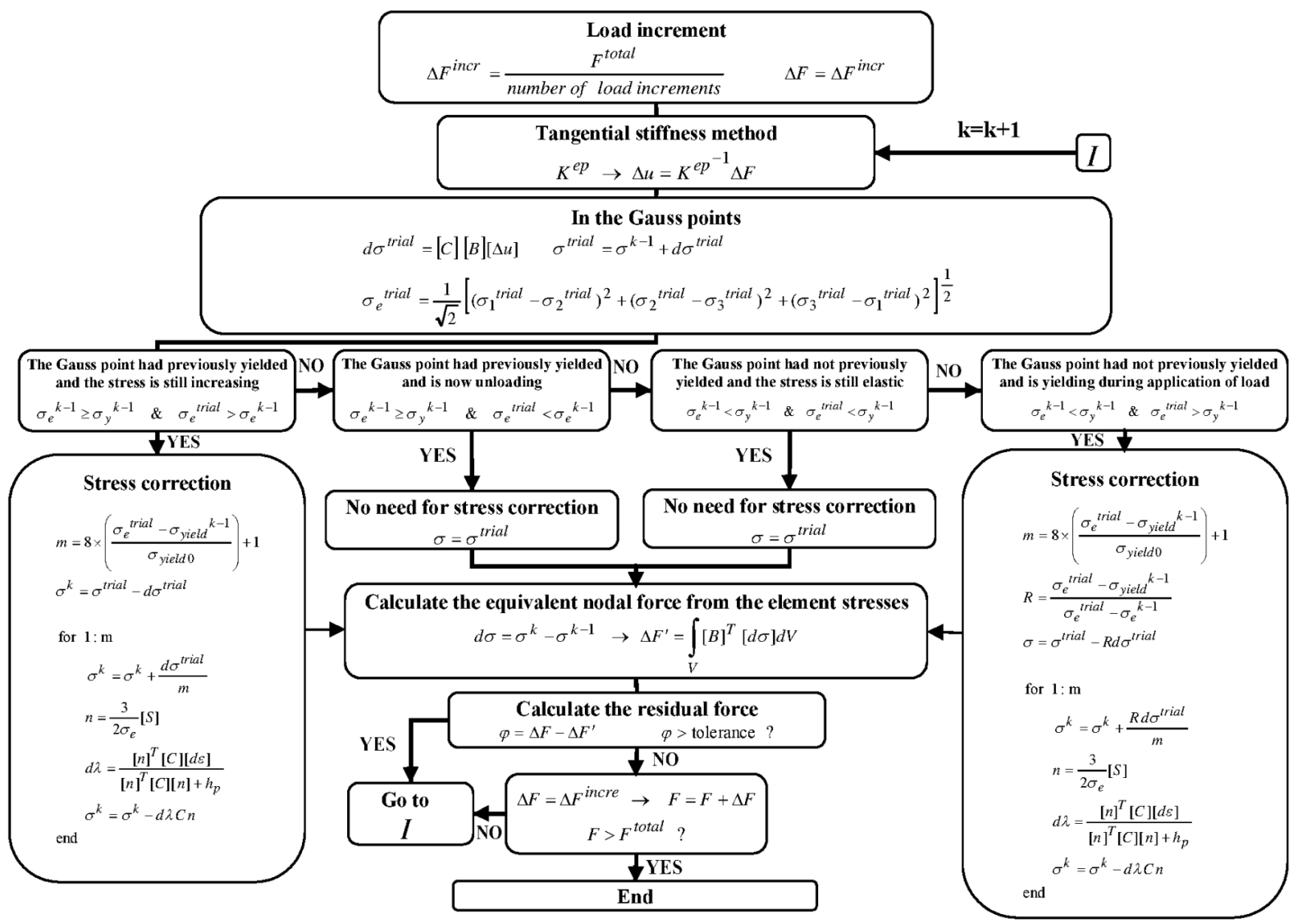

Fig. 2. Flow chart of the algorithm for the elastic-plastic analysis 


\section{Mechanical behaviour of FGM}

It is assumed that the functionally graded metal-ceramic composite is locally isotropic and follows the von Mises yield criterion. The three important material properties for elastic-plastic analysis are the elastic modulus $E(r)$, the initial yield stress $\sigma_{y 0}(r)$, and the tangent modulus $H(r)$. These properties can be calculated using the modified rule of mixtures for composites (Suresh and Mortensen, 1998). Note that the modified rule of mixtures is appropriate for modeling of isotropic materials

$$
\begin{aligned}
& E=\left[\left(1-f_{c}\right) E_{m} \frac{q+E_{c}}{q+E_{m}}+f_{c} E_{c}\right]\left[\left(1-f_{c}\right) \frac{q+E_{c}}{q+E_{m}}+f_{c}\right]^{-1} \\
& \sigma_{y 0}=\sigma_{y 0 m}\left[\left(1-f_{c}\right)+\frac{q+E_{m}}{q+E_{c}} \frac{E_{c}}{E_{m}} f_{c}\right] \\
& H=\left[\left(1-f_{c}\right) H_{m} \frac{q+E_{c}}{q+H_{m}}+f_{c} E_{c}\right]\left[\left(1-f_{c}\right) \frac{q+E_{c}}{q+H_{m}}+f_{c}\right]^{-1} \\
& h_{p}=\frac{E H}{E-H}
\end{aligned}
$$

where the subscripts $c$ and $m$ indicate the ceramic and metal material, respectively. The volume fraction of ceramic particles is denoted by $f_{c}$, and $q$ is the ratio of the stress to strain transfer, where $\sigma_{c}, \varepsilon_{c}$ and $\sigma_{m}, \varepsilon_{m}$ are the average stresses and strains in ceramic and metal, respectively (see Fig. 3)

$$
q=\frac{\sigma_{c}-\sigma_{m}}{\left|\varepsilon_{c}-\varepsilon_{m}\right|} \quad 0<q<\infty
$$

(a)

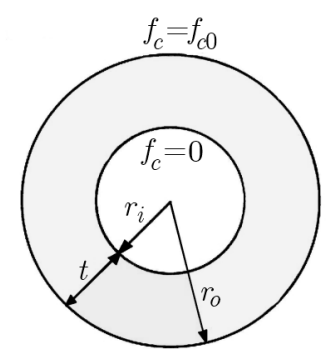

(b)

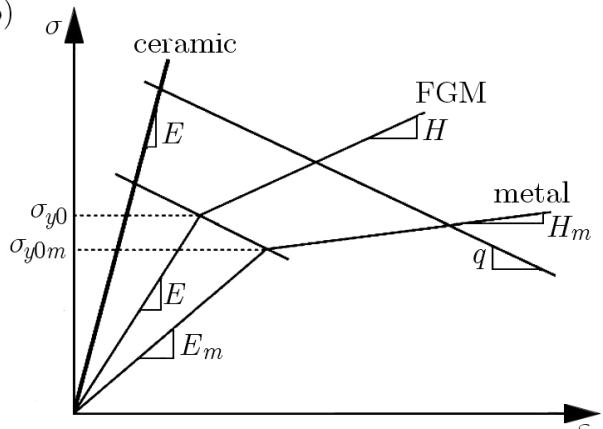

Fig. 3. (a) Schematic representation of a thick FGM vessel (rotating disk) with internal radius $r_{i}$ and external radius $r_{o}$. (b) Schematic representation of the modified rule of mixtures used to estimate the behaviour of the ceramic particle-reinforced metal composite

The empirical parameter $q$ depends on many factors including material composition, microstructural arrangements and internal constraints. For example, $q \rightarrow \infty$ if the constituent elements deform identically in the loading direction, while $q=0$ if the constituent elements experience the same stress level. In the present analysis, the ceramic particle reinforcement is assumed to have a volume fraction that varies from 0 at the inner radius $r_{i}$ to $f_{c 0}$ at the outer radius $r_{o}$ according to the following relationship

$$
f_{c}(r)=f_{c 0}\left(\frac{r-r_{i}}{r_{o}-r_{i}}\right)^{n}
$$

where $n$ is the reinforcement distribution exponent $n=0$ denotes uniformly-reinforced metal-ceramic). The material properties for each constituent phase are listed in Table 1. The parameter $q$ may be approximated by calibration of experimental data from tensile tests performed on monolithic composite specimens. For example, a value of $q=4.5 \mathrm{GPa}$ is used for a $\mathrm{TiB} / \mathrm{Ti}$ FGM (Carpenter et al., 1999), whereas Poisson's ratio is taken constant and equal to 0.3. 
Table 1. Material properties (Jin et al., 2003)

\begin{tabular}{|l|c|c|c|}
\hline Materials & $\begin{array}{c}\text { Young's modulus } \\
{[\mathrm{GPa}]}\end{array}$ & $\begin{array}{c}\text { Yield stress } \\
{[\mathrm{MPa}]}\end{array}$ & $\begin{array}{c}\text { Tangent modulus } \\
{[\mathrm{GPa}]}\end{array}$ \\
\hline \hline $\mathrm{Ti}$ & 107 & 450 & 10 \\
\hline $\mathrm{TiB}$ & 373 & & \\
\hline
\end{tabular}

\section{Results}

In this Section, we study three cases, a cylindrical and spherical pressure vessel as well as a rotating disk made of FGMs. The elastic-plastic isogeometric analysis code is written in MATLAB (2008). The cylinder (disk) B-spline model includes 50 control points (10 points in the $r$-direction and 5 points in the $z$-direction), and the assumed knot vectors are $\boldsymbol{\Xi}=[0,0,0,0,0.142,0.285,0.428,0.571,0.714,0.857,1,1,1,1]$ and $\mathbf{H}=[0,0,0,0,0.5,1,1,1,1]$ (cubic NURBS). In all the cases, the second order Gauss quadrature is used for numerical integrations (156 Gauss points in the $r$-direction and 8 Gauss point in the $z$-direction). The knot vectors and the number of control points in the sphere model is similar to that in the cylinder model. The geometries in this study are modeling with a single patch (the patch for the surface is the domain $\left[\xi_{1}=0, \xi_{n+p+1}=1\right] \times\left[\eta_{1}=0, \eta_{n+p+1}=1\right]$ ), and we have no need to assemble the stiffness matrices and force vectors. By removing the assemble step, we reduce the overall analysis time by about $10 \%$ (at the same number of degrees-of-freedom in the isogeometric method and FEM).

To verify the accuracy of the isogeometric analysis, finite element analyses have been performed using the commercial finite element code ABAQUS (Karlsson, Hibbitt, Sorensen Inc., 2008). The conventional method of modelling FGMs in the software is to subdivide the thick wall into several thin layers with equal thickness. This method of modelling leads to discontinuity in the mechanical properties of FGM materials and is both difficult and time-consuming. Setoodeh et al. (2008) proposed a new approach for analysing the FGM material in the elastic zone without the need for dividing the thickness into thin strips. They applied a virtual temperature distribution in the cylinder wall using the facility available within the software to assign continuously variable properties across the wall thickness and then created a one-to-one relationship between the temperature and mechanical properties. The correlation of the distribution of temperature and mechanical properties of the FGM material allowed one tp model the variation of FGM properties in the cylinder. Note that the conductivity factor and other thermal parameters are set to zero, and the temperature does not change during analysis. Indeed, the analysis is solely mechanical and no thermomechanical examination is carried out. This method allows the analysis of the elastic-plastic FGM cylindrical and spherical vessels as well as the rotating disk. The three-dimensional 8-noded linear coupled temperature displacement family of finite elements in ABAQUS has been used to model the cylinder. Sensitivity analysis of the mesh has also been performed to ensure the results remained insensitive to the element size.

In order to evaluate the isogeometric analysis code, a set of results from finite element calculations and the VMP method (Haghpanah Jahromi et al., 2009) obtained for the plane strain conditions have been compared with the results obtained from the isogeometric analysis for the FGM cylindrical vessel subjected to autofrettage internal pressure $100 \mathrm{MPa}$ (Fig. 4). The results indicated that the isogeometric analysis method has some influence on the accuracy. Figure 5 illustrates the distribution of von Mises stresses across the thickness in the cylindrical vessel subjected to internal pressure $300 \mathrm{MPa}$ with $n=2$ and different reinforcement distribution coefficients $f_{c 0}$. The results show that because $E_{c} / E_{m}>1$, an increase in $f_{c 0}$ elevates von Mises stress in the outer surface, and the plastic region decreases in the inner surface. 


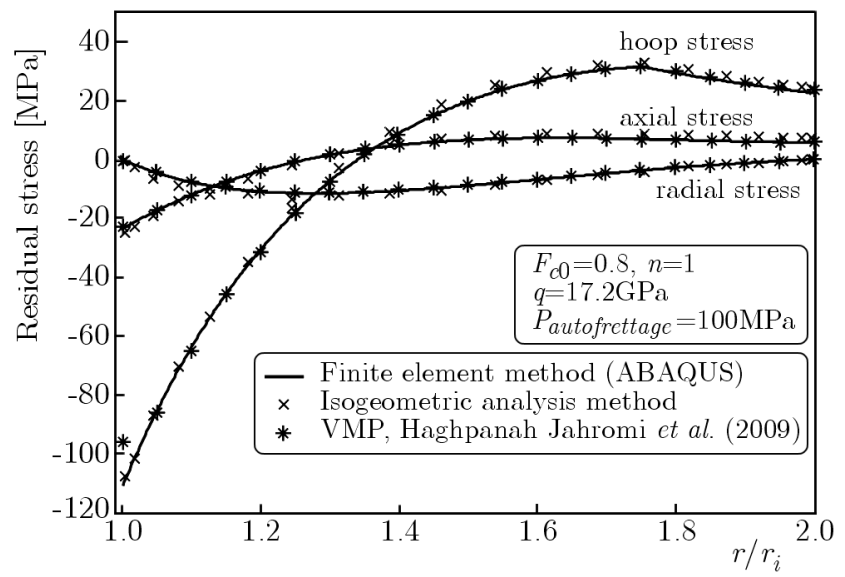

Fig. 4. Comparison of the VMP method (Haghpanah Jahromi et al., 2009) with the finite element analysis. The results show the residual stresses in the autofrettaged vessel. In this calculation: $E_{m}=56 \mathrm{GPa}, E_{c}=20 \mathrm{GPa}, \sigma_{y 0 m}=106 \mathrm{MPa}, H_{m}=12 \mathrm{GPa}$ and $\nu_{m}=\nu_{c}=0.25$. The vessel undergoes a plane-strain condition

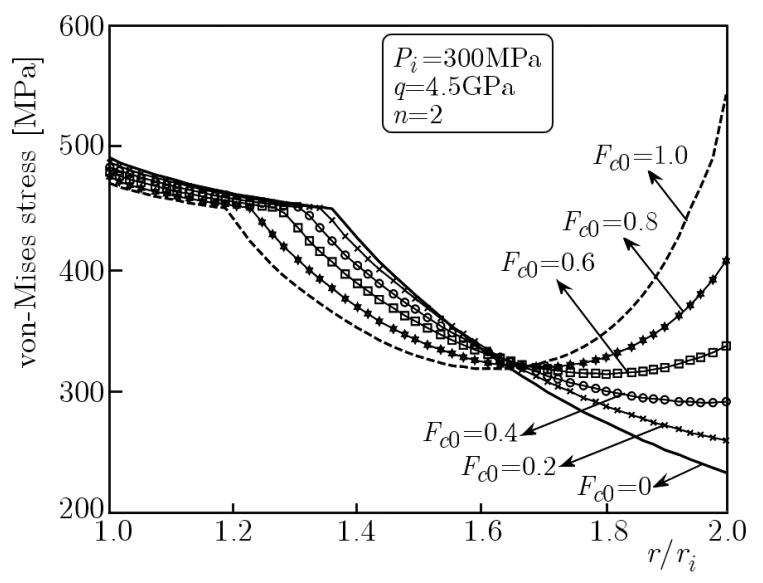

Fig. 5. Von Mises stress along the thickness in the FGM cylindrical vessel subjected to internal pressure $300 \mathrm{MPa}$ with $q=4.5 \mathrm{GPa}$ and $n=2$ for different $f_{c 0}$. The vessel has $t / r_{i}=1$ and plane-stress condition (properties listed in Table 1)

For the spherical vessel subjected to internal pressure of $600 \mathrm{MPa}$, the results obtained from the isogeometric analysis method have been compared with the finite element results. Excellent agreement is observed as shown in Fig. 6. For the purpose to investigate the effect of $n$ on the initiation of yielding, we introduce two parameters $P_{v 1}$ (pressures corresponding to the initiation of yielding at the inner radius) and $P_{v 2}$ (pressures corresponding to the initiation of yielding at the outer radius). Figure 7 shows that $P_{v 1}$ and $P_{v 2}$ decrease with growing $n$. Also, in the cylinder subjected to $P_{v 2}$, by increasing $n$, the plastic region gradually spreads from the inner surface. Note that by increasing $n$, the metal properties dominate overcoming the ceramic properties and, therefore, the plastic behaviour of the material becomes more evident.

Similar to the previous cases, the excellent agreement of the isogeometric analysis with the finite element predictions of elastic-plastic stresses for the rotating disc is shown in Fig. 8. The distribution of von Mises stresses across the thickness in the disk rotating at different angular velocities with $n=2$ and $f_{c 0}=0.8$ is presented in Fig. 9. The results clearly indicate that the growth of the plastic zone across the thickness is initiated from both sides of the disc. In this study, density of metal is $\rho_{m}=4420 \mathrm{~kg} / \mathrm{m}^{3}$ and density of ceramic is $\rho_{c}=2000 \mathrm{~kg} / \mathrm{m}^{3}$. 


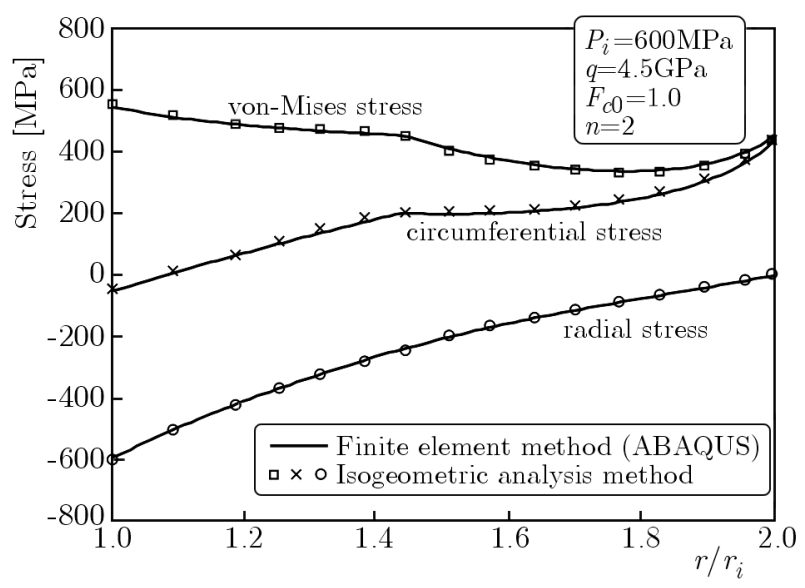

Fig. 6. Comparison with the finite element method. The results show the stress components in the spherical vessel with $f_{c 0}=1$ and $n=2$ subjected to internal pressure $600 \mathrm{MPa}$. In this calculation $q=4.5 \mathrm{GPa}$. The vessel has $t / r_{i}=1$ (properties listed in Table 1 )

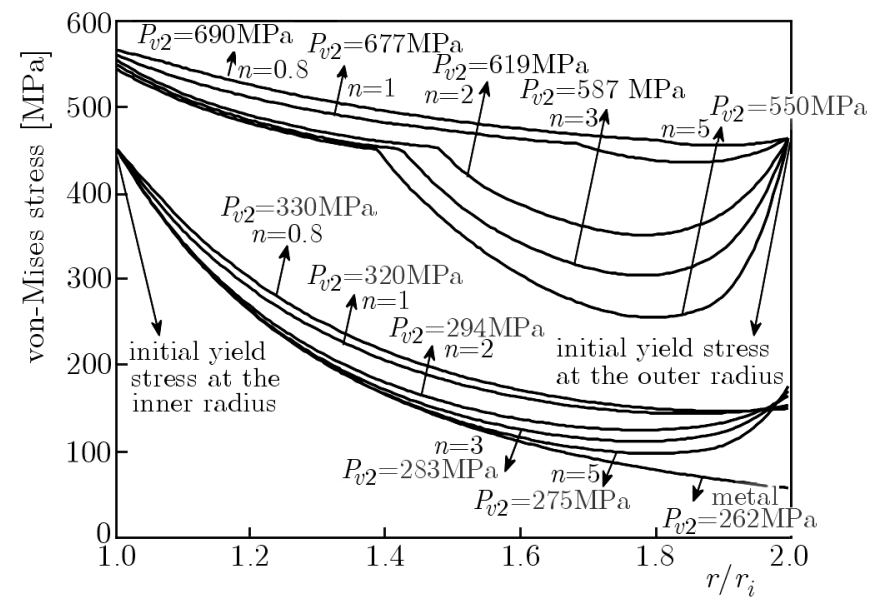

Fig. 7. Von Mises stress along the thickness in the FGM spherical vessel subjected to $P_{v 1}$ and $P_{v 2}$ with $f_{c 0}=1$ and different $n$. In this calculation $q=4.5 \mathrm{GPa}$. The vessel has $t / r_{i}=1$ (properties listed in Table 1)

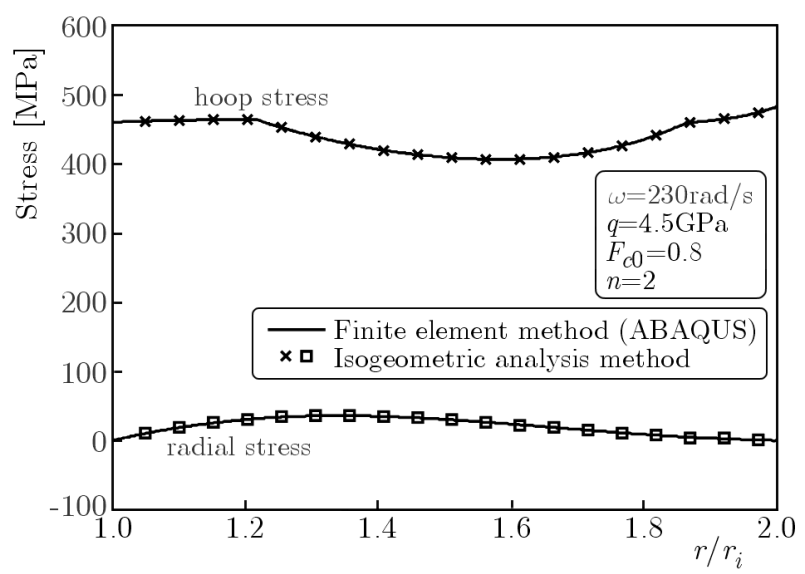

Fig. 8. Comparison with the finite element method. The results show the stress components in the rotating disk with $f_{c 0}=0.8$ and $n=2$ at the angular velocity $\omega=230 \mathrm{rad} / \mathrm{s}$. In this calculation $q=4.5 \mathrm{GPa}$. The disk has $t / r_{i}=1$ (properties listed in Table 1) 


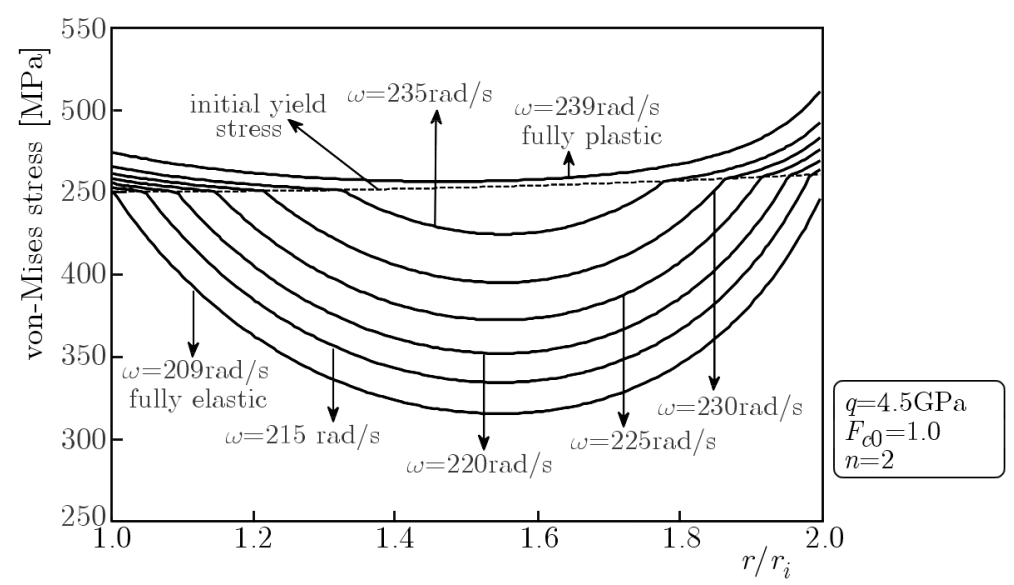

Fig. 9. Von Mises stress along the thickness in the FGM rotating disk at different angular velocities with $f_{c 0}=0.8$ and $n=2$. In this calculation $q=4.5 \mathrm{GPa}$. The disk has $t / r_{i}=1$ (properties listed in Table 1 )

\section{Conclusion}

Using the isogeometric analysis method, elastic-plastic stress distributions in a cylindrical and spherical pressurized vessels and rotating disks made of an FGM material have been determined. As expected, this approach to the plasticity problem is computationally cost effective and results in a much smaller system of equations to solve. Finite element analysis of the problem using ABAQUS commercial code has been used for verification of the isogeometric method. The numerical analysis within the software has been performed by the application of a "virtual thermal load" that enabled continuous variation of the material behaviour through the wall thickness. The analysis results obtained in this work also indicate the possibility of formation and growth of a plastic region within the wall thickness from the external surface of the FGM vessels or rotating disks whereas in cylindrical (spherical) vessels and rotating disks made of homogeneous materials, the plasticity essentially starts from the inner surface.

\section{References}

1. Bayat M., Saleem M., Sahari B.B., Hamouda A.S.M., Mahdi E., 2008, Analysis of functionally graded rotating disks with variable thickness, Mechanics Research Communications, 35, 283-309

2. Carpenter R.D., Liang W.W., Paulino G.H., Gibeling J.C., Munir Z.A., 1999, Fracture testing and analysis of a layered functionally graded $\mathrm{Ti} / \mathrm{TiB}$ beam in 3-point bending, Materials Science Forum, 308, 837-842

3. Chakraborty A., Gopalakrishnan S., Reddy J., 2003, A new beam finite element for the analysis of functionally graded materials, International Journal of Mechanical Sciences, 45, 519-539

4. Chen Y.Z., Lin X.Y., 2008, Elastic analysis for thick cylinders and spherical pressure vessels made of functionally graded materials, Computational Materials Science, 44, 581-587

5. Cottrell J.A., Hughes T.J.R., Bazilevs Y., 2009, Isogeometric Analysis: Toward Integration of CAD and FEA, John Wiley, U.K.

6. Dai H.L., Fu Y.M., Dong Z.M., 2006, Exact solutions for functionally graded pressure vessels in a uniform magnetic field, International Journal of Solids and Structures, 43, 5570-5580

7. Durodola J.F., Atтia O., 2006, Deformation and stresses in functionally graded rotating disks, Composites Science and Technology, 60, 987-995 
8. Figueiredo F., Borges L., Rochinha F., 2008, Elasto-plastic stress analysis of thick-walled FGM pipes, AIP Conference Proceedings, 147-152

9. Haghpanah Jahromi B., Ajdari A., Nayeb-Hashemi H., Vaziri A., 2010, Autofrettage of layered and functionally graded metal-ceramic composite vessels, Composite Structures, 92, 1813-1822

10. Haghpanah Jahromi B., Farrahi G.H., Maleki M., Nayeb-Hashemi H., Vaziri A., 2009, Residual stresses in autofrettaged vessel made of functionally graded material, Engineering Structures, 31, 2930-2935

11. Haghpanah Jahromi B., Nayeb-Hashemi H., Vaziri A., 2012, Elasto-plastic stresses in a functionally graded rotating disk, ASME Journal of Engineering Materials and Technology, 134, 21004-21015

12. Hassani B., Tavakkolin S.M., Moghadama N.Z., 2011, Application of isogeometric analysis in structural shape optimization, Scientia Iranica, 18, 864-852

13. Hughes T.J.R., Cottrell J.A., Bazilevs Y., 2005, Isogeometric analysis: CAD, finite elements, NURBS, exact geometry and mesh refinement, Computer Methods in Applied Mechanics and Engineering, 194, 4135-4195

14. Jahed H., Dubey R.N., 1997, An axisymmetric method of elastic-plastic analysis capable of predicting residual stress field, Journal of Pressure Vessel Technology, 119, 264-273

15. Jahed H., Farshi B., Bidabadi J., 2005, Minimum weight design of inhomogeneous rotating discs, International Journal of Pressure Vessels and Piping, 82, 35-41

16. Jahed H., FArshi B., Karimi M., 2006, Optimum autofrettage and shrink-fit combination in multi-layer cylinders, Journal of Pressure Vessel Technology, 128, 196-201

17. Jahed H., Shirazi R., 2001, Loading and unloading behaviour of a thermoplastic disc, International Journal of Pressure Vessels and Piping, 78, 637-645

18. Jin Z.H., Paulino G.H., Dodds JR R.H., 2003, Cohesive fracture modelling of elastic-plastic crack growth in functionally graded materials, Engineering Fracture Mechanics, 70, 1885-1912

19. Karlsson, Hibbitt, Sorensen, 2008, ABAQUS/CAE, v. 6.8-1.

20. Owen D.R.J., Hinton E. 1980, Finite Elements in Plasticity: Theory and Practice, Pineridge Press, U.K.

21. Sadeghian M., Ekhteraei H., 2011, Axisymmetric yielding of functionally graded spherical vessel under thermo-mechanical loading, Computational Materials Science, 50, 975-981

22. Setoodeh A., Kalali A., Hosseini A., 2008, Numerical analysis of FGM plate by applying virtual temperature distribution, Proceedings of 7th Conference of Iranian Aerospace Society, Tehran

23. Suresh S., Mortensen A., 1998, Fundamentals of Functionally Graded Materials, IOM Communications Ltd.

24. You L.H., Zhang J.J., You X.Y., 2005, Elastic analysis of internally pressurized thick-walled spherical pressure vessels of functionally graded materials, International Journal of Pressure Vessels and Piping, 82, 374-345 